\title{
Gravitational waves and multimessenger astronomy
}

\author{
Fulvio Ricci ${ }^{1,2, a}$ \\ ${ }^{1}$ Dipartimento di Fisica, Università di Roma La Sapienza, Italy \\ ${ }^{2}$ INFN Sezione di Roma, Rome, Italy
}

\begin{abstract}
It is widely expected that in the coming quinquennium the first gravitational wave signal will be directly detected. The ground-based advanced LIGO and Virgo detectors are being upgraded to a sensitivity level such that we expect to be measure a significant binary merger rate.

Gravitational waves events are likely to be accompanied by electromagnetic counterparts and neutrino emission carrying complementary information to those associated to the gravitational signals. If it becomes possible to measure all these forms of radiation in concert, we will end up an impressive increase in the comprehension of the whole phenomenon.

In the following we summarize the scientific outcome of the interferometric detectors in the past configuration. Then we focus on some of the potentialities of the advanced detectors once used in the new context of the multimessenger astronomy.
\end{abstract}

\section{From the first to the second generation of GW detectors}

Since 1960 various laboratory experiments have been carried on to detect gravitational waves $(\mathrm{GW})$. The detector technique evolved from the simpler room resonant detectors of $\mathrm{J}$. Weber to the cryogenic ones and at present the field is dominated by the operation of the giant interferometric devices installed in Europe, Japan and USA. The device sensitivity and its bandwidth is increased progressively expanding the search domain on a larger variety of GW signals. However, since the Weber's epoch it was clear that this experimental challenge requires the coordinate effort of more than one detector installed in different Earth locations.

The first attempt to observe a gravitational wave signal using a world wide network was in 1986 the coincidence experiment of the three cryogenic resonant-mass detectors set up by the universities of the Louisiana state, Rome and Stanford. [1]. In more recent years the International Gravitational Event Collaboration, derived upper limit on the event rate of GW bursts using observational data of the resonant detectors in operation taken during almost three years of run [2]. Nowadays, the network of the GW advanced detectors is based on all the data produced by the interferometers of the GEO [3], LIGO [4] and Virgo [5] allowing for maximal sky coverage and permitting the transient source location by triangulation. In the near future we expect that the KAGRA instrument [6], installed in Japan, will join the network improving significantly the capability of the sky localisation of the GW event.

GEO600 in Ruthe, Germany is a $600 \mathrm{~m}$ long arm interferometer in a dual-recycled configuration, i.e. it includes both power and signal recycling. It is equipped with a homodyne

\footnotetext{
a e-mail: fulvio.ricci@roma1.infn.it
} 
detection system and quantum squeezing. The instruments results from an international collaboration of Germany and United Kingdom. It does not include Fabry-Perot cavities in its arms, as it is the case for LIGO and Virgo, and its best sensitivity corresponds to an amplitude spectral density $\sim 3 \times 10^{-21} 1 / \sqrt{\mathrm{Hz}}$ in a $2 \mathrm{kHz}$ bandwidth around $1 \mathrm{kHz}$.

Advanced LIGO are two interferometers, now in the commissioning phase, located in Hanford (Washington state, USA), and in Livingston (Louisiana state, USA). Groups at Caltech and MIT are operating these $4 \mathrm{~km}$ long arm instruments. In the past years LIGO achieved a sensitivity of about $2 \times 10^{-23} 1 / \sqrt{\mathrm{Hz}}$ at $150 \mathrm{~Hz}$.

The Virgo detector is installed at Cascina (Italy) and the European Gravitational Wave consortium of the CNRS-France and INFN-Italy is managing the site. The instrument is operated by an international collaboration collaboration of five european nation, which includes France, Italy, Nederland, Hungary and Poland. The facility has perpendicular $3 \mathrm{~km}$ long arms and took data during several scientific runs achieving a sensitivity of $6 \times 10^{-23} 1 / \sqrt{\mathrm{Hz}}$ in a broad bandwidth around $200 \mathrm{~Hz}$.

In 2008, the installation of the advanced LIGO (aLIGO) detectors was started and three years later the advanced Virgo project followed. The new instruments will use a higher power laser to reduce the shot noise at high frequencies, the signal recycling and fused silica monolithic suspensions for the last stage of mirror suspension, to reduce the thermal noise, which limits the sensitivity at low and mid frequencies. The advanced detectors have a designed sensitivity about ten times better than the initial detectors and thus will be capable of observing sources from a spatial volume a thousand times larger.

Both the advanced LIGO detectors at Hanford and Livingston are in the commissioning phase and we will begin operating in 2015 , gradually achieving the final design sensitivity with many plausible detections.

The Virgo detector will join the network one year later providing localization.

Deep under the mountain of the Kamioka mine in Kamioka-cho, Gifu-prefecture of Japan, the Kamioka Gravitational Wave detector (KAGRA) is under construction. It is now likely to enter operation in 2018/2019. To achieve the required sensitivity, several advanced techniques will be employed such as a low-frequency vibration-isolation system, cryogenic mirrors, a resonant side band extraction method.

Since the detection probability increases with the number of detectors, it is very likely that gravitational wave detections will happen in the next quinquennium, starting the new era of gravitational wave astronomy. These signals produced by the coherent motion of large masses will permit to probe the properties of the gravitational interaction. The GW detection will open the study of gravitational field propagation, which is described by the Einstein General Relativity, a theory based on the principle of local invariance with respect to arbitrary transformations of the coordinate systems. This invariance is extended to the description of the force fields as it happen for example in the case of the electroweak interactions, providing a conceptual basis for the unification of gravity with the other elementary forces.

In addition to the impact on fundamental physics, the first direct detection of gravitational wave signals will open a new era for Astronomy, as we will try to demonstrate in a following section.

\section{The legacy of the first generation}

Here we will try to summarise the scientific results obtained using the km-size interferometers of the first generation. For convenience we will follow the classic division between the searches for continuous signals and those related to the transients. 


\subsection{Continuous signals}

The way to search for continuous GW signals depends on how much about the source is known. At present, about 2000 neutron stars in our Galaxy have been identified by their radio, X-ray or $\gamma$ ray emissions. The effort to detect a GW signal emitted by one of these sources is called target search. However, our Galaxy is estimated to contain roughly 100 million neutron stars, so the all-sky search for continuous GWs might detect new ones and tell us about their structure.

Aiming at signals potentially emitted by isolated radio pulsars and incorporating phase information deduced by radio observations, the target search was able to set limits on the GW metric perturbation $h_{c}$ as low as a few $10^{-24}$, limits which could be translated into star ellipticities $\epsilon<10^{-5}$ for the four closest pulsars, thus starting to probe plausible astrophysical values.

Using the data of the last run of Virgo, thanks to the good low-frequency sensitivity, an upper limit on the emission by the Vela pulsar [7] was obtained on the assumption that the phase of the GW signal can be modeled on the radio emission, and that spin axis and wave polarization angles are known. The limit lyes in the interval $h_{c}<(1.9-2.2) \times 10^{-24}$, thus beating the spin down limit $h_{c, \max }=3.3 \times 10^{-24}$ derived from the pulsar period evolution measured using the electromagnetic emission of the pulsar.

The targeted search was extended to 116 known millisecond and young pulsars using LIGO and Virgo data allowing to set a limit on the Crab GW emission, a factor seven below the spin-down limit, which means less than $2 \%$ of the available spin-down power. For several of the other young pulsars, the limits are only slightly above the spin-down limits. The best results were obtained for J1603-720, with a limit $h_{c}<2.3 \times 10^{-26}$, and for J2124-3358, with a limit $\epsilon<7 \times 10^{-8}[8]$.

The all-sky search for periodic signals based on LIGO and Virgo data [9], has been carried on in the frequency band of $50-800 \mathrm{~Hz}$ assuming a first derivative of the emission frequency lying in the interval $6 \times 10^{-9}-0 \mathrm{~Hz} / \mathrm{s}$ and we set a limit of $h_{c}<3.8 \times 10^{-24}$ for all polarizations and sky locations.

A different category of continuos signals is the GW stochastic background of both astrophysical and cosmological origins. In this case, the detection technique is based on the cross-correlation of the outputs of different instruments. During the LIGO run S5 and the first Virgo Science run, an upper limit on the normalized energy density of GW stochastic background $h_{o}^{2} \Omega_{G W}$ at $150 \mathrm{~Hz}$ was set under the hypothesis of an isotropic signal distribution [11],

$$
h_{o}^{2} \Omega_{G W}<6.9 \times 10^{-6} \text { at } 150 \mathrm{~Hz}
$$

and at higher frequencies [12]

$$
h_{o}^{2} \Omega_{G W}<0.16\left(\frac{f}{900 \mathrm{~Hz}}\right)^{3} \text { for } 600 \mathrm{~Hz}<f<1000 \mathrm{~Hz} .
$$

\subsection{The transient signals}

In this category we distinguish between generally unmodeled signals (GW burst) and those of the binary system coalescence with defined GW waveform.

In the latter case the analysis of LIGO S6 data and Virgo second and third science run data provides for the low-mass binary systems the best results so far [13]. Assuming that the total mass of the binary system $M_{t o t}$ is in the interval 2-25 solar masses the search had a distance 
limit for events associated to a neutron star-neutron star system (NS-NS) as far as $40 \mathrm{Mpc}$, a distance averaged over the observation time and over source sky location and orientation. This corresponds to set a limit for the event rate per unit volume of the Universe

$$
R_{N S-N S}<1.3 \times 10^{-4} \mathrm{yr}^{-1} \mathrm{Mpc}^{-3}
$$

In the case of higher mass system including one or two black holes $(\mathrm{BH})$ we end up with

$$
R_{N S-B H}<3.1 \times 10^{-5} \mathrm{yr}^{-1} \mathrm{Mpc}^{-3}
$$

and

$$
R_{B H-B H}<6.4 \times 10^{-6} \mathrm{yr}^{-1} \mathrm{Mpc}^{-3} .
$$

The same data allowed to improve limits on binary black hole events, with an analysis which exploited all phases (inspiral, merger, ringdown) of the signal [14]: for $M_{\text {tot }}$ ranging in the interval of 25-100 solar masses, the search achieved the best distance value of $300 \mathrm{Mpc}$ setting the limit

$$
R_{B H-B H}<3.3 \times 10^{-7} \mathrm{yr}^{-1} \mathrm{Mpc}^{-3}
$$

for systems with non-spinning components in the interval 19-28 solar masses.

For signals of unknown shape, it is generally convenient to define the strength of the detected signal in terms of the root sum square (rss) amplitude $h_{r s s}$ of the signal $h(t)$.

The joint LIGO S6 run, and the second and third Virgo runs allowed to achieve the best results [15]: over a total observation time of 207 days, for signals shorter than $1 \mathrm{~s}$ lying in the $64-5000 \mathrm{~Hz}$ frequency band, we set an upper limit on the rate of 1.3 events/year in the range of amplitude spectral density sensitivity of $5 \times 10^{-22}-1 \times 10^{-20} \mathrm{~Hz}^{-1 / 2}$. For a burst emission in the center of our Galaxy $(\sim 10 \mathrm{kpc})$, these values allow a detect with $50 \%$ efficiency, if the sources emit an energy of about $2.2 \times 10^{-8}$ solar masses, a value not far from the prediction of the modern models of a supernova explosion [16, 17].

\section{Advanced detectors and multimessenger astronomy}

The technological progress achieved in the construction of the new generation of advanced detectors, and the knowledge gained in astrophysical sources and in data analysis techniques promises that the next years will generate exciting progress in the comprehension of several astrophysical phenomena.

Compact binary coalescence is one of the most important sources for ground-based interferometers and the waveform of the related GW signal at the inspiral phase is fairly well understood theoretically. The expected detectable distance in the advanced detector era for NS-NS binaries is around 150-200 Mpc and the most probable event rate is around $40 \mathrm{yr}^{-1}$ for advanced LIGO and Virgo.

The signal is dominated by the inspiral phase, well described by the post-newtonian approximation, so that standard matched filtering allows to search for events in the detector noise. However the most interesting information is hidden in the final phase near the merger when the stars are colliding, and the corresponding part of the GW signal brings the information on the equation of state of the nuclear matter. In fact, during the last stages of inspiral, the Newtonian tidal field of one component induce a quadrupole moment in the other. The effect depends on the tidal deformability of the massive star in a way that is determined by its equation of state. The neutron stars deformation has an influence on the orbital motion, in particular the phase, which up to a factor of two is also the phase of the emitted gravitational wave signal. In particular, it has been shown [18] that, in a realistic data analysis setting, quantitative information about the size of the tidal deformability at a given 
reference mass can be obtained with a $2-\sigma$ statistical uncertainty of $\sim 10 \%$ after a few tens of detections, and hypothesis ranking will be able to distinguish between a hard, moderate, and soft equation of state.

Direct GW measurement of a coalescing binary provides a distance-ladder-independent measure of the luminosity distance $D_{L}$ to a source. While $D_{L}$ may be well measured, the source redshift cannot be inferred directly from the GW signal. An independent measure of the event redshift is therefore required to measure cosmological parameters with GW events. If an electromagnetic (EM) signature is detected, spectroscopic observations of the event or the galaxy hosting the EM event should be obtainable. Thus, we can use inspirals as cosmological probes when we associate the gravitational event with an electromagnetic counterpart.

This is the first example of potential outcome of a multimessenger approach providing a new tool for precision cosmology, but there are several other benefits of joint GW and EM observations. EM localization of a GW transient event reduces significantly the parameter space of search templates, and correspondingly increasing the observed signal-to-noise ratio (SNR). Likewise, an identification drastically cuts down the search range in time, reducing the threshold signal-to-noise required for confident detection.

Many astrophysical sources, the majority of which originate from cataclysmic events, are expected to produce both GWs and high energy neutrinos (HEN). While GWs are linked to the dynamics of the bulk motion of the source progenitor, HENs trace the interactions of accelerated protons (and possibly heavier nuclei) with matter and radiation in and around the source. Stellar core collapse during a supernovae (SN) explosion releases enough gravitational binding energy in less than one second, involving mass asymmetric motion to generate strong gravitational-wave signals.

Long-soft $\gamma$ ray bursts, which represent the majority of $\gamma$ ray events (GRB) events, have been related to massive stellar explosions [19]. However, neither all SNe, nor even all SNe of Type Ibc produce GRBs. The degree of differential rotation in the collapsing iron core of massive stars when they die may be what makes the difference. The detection of GWs from a SN strongly constrains the rotation of the collapsed core. A SN close enough to be a strong GW source, which is also a strong neutrino source, with the information associated to the photon emission become a powerful tool to study the physics of the matter at nuclear densities.

The study of the origin of short-hard $\gamma$ ray bursts (SHB) has shown an indirect evidence that they are associated to an older stellar population, driving the hypothesis that binary mergers $(N S-N S$ or $N S-B H)$ are the short $\gamma$ ray progenitors. However, a number of other origins remain viable: a concurrent GW inspiral event in the same place and time will solve the enigma. In the standard picture, the mechanism responsible for the enormous EM flux in the prompt emission and in the afterglow of a SHB is the dissipation (via internal and external shocks) of bulk kinetic into highly relativistic particles; the particles are accelerated to a nonthermal energy distribution in a relativistically expanding fireball ejected by the GRB central engine. The internal or external shocks accelerate protons that interact with the gamma rays and/or other protons inside the fireball, producing charged pions and kaons that subsequently decay into high energy neutrinos (HEN). While $\gamma$ ray and HEN emissions from GRBs are related to the mechanisms driving the relativistic outflow, GW emission is closely connected to the central engine and hence to the progenitor of the GRB. In addition to the information extracted from the GW signal shape, the difference between the times of arrival of GW and HEN signals provides an important information on the emission mechanism: the detection of a HEN signal prior to a GW one may indicate that the strongest GW emission from the source is not connected to the onset of the activity of the central engine that one might expect from core-collapse models. 
To complete the picture, we note also that the emission of short GRBs and the related HEMs is beamed and, hence we can expect also cases in which GWs will be observed from such sources without an observed GRB or HEN signal. However, an orphan afterglow, macronovae or radio flares might be observed in these cases. In conclusion, combining information from all these independent detectors can provide original ways of constraining the physical processes driving the source.

Finally we have to cite that on the order of 20 highly magnetized neutron stars are observed in our Galaxy as soft $\gamma$ repeaters (SGR) and anomalous X-ray pulsars (AXP). They emit short bursts of X-rays and soft $\gamma$ rays and sometimes giant flares or storms of bursts. The energy release may excite neutron star crustal f-modes in the $\mathrm{kHz}$ region emitting GW signals with the typical ring down signature. Torsional (shear) s-mode core-quakes may release significantly more GW energy [20].

A upper limit on the GW emission from six magnetars was set already using LIGO and Virgo data [21] and we expect a factor 100 better sensitivity (in emitted energy) with advanced detectors.

\section{Conclusion}

The global advanced detector network, which includes the two LIGO detectors, GEOHF, Virgo, KAGRA will be operative in the next quinquennium and possibly LIGO-India will join in the future. The first science run of LIGO is approaching and next year Virgo will be put in operation. The network enables more confident detection by requiring the same or similar signal in widely separated instruments. More important, a network locate the sources in the sky, since individual detectors have essentially no ability determine the source direction: the network acts as a gravitational-wave interferometer, determining source direction through timing and amplitude information of a wave detected coherently across a global baseline. Good source localisation allows one to look for other forms of radiation coming from that location, nearly simultaneously with the GWs: optical, radio, $\mathrm{X}$-ray or gamma electromagnetic radiation, or neutrinos. Such multi-messenger signals may be expected from sources containing ordinary atomic or nuclear matter.

Thus, we expect that the searches undertaken by advanced detectors will be indeed likely capable of detecting, constraining, or excluding several existing astrophysical models, contributing in the future to a significant progress in Astronomy.

\section{References}

[1] E. Amaldi et al., Astron. and Astrophys. 216, 325 (1989)

[2] P. Astone et al., Phys. Rev. D68, 022001 (2003)

[3] http://www.geo600.org

[4] http://www.ligo.caltech.edu

[5] F. Acernese et al., Class. Quantum Grav. 32, 024001 (2015)

[6] http://gwcenter.icrr.u-tokyo.ac.jp/en

[7] J. Abadie et al., Astrophys. J. 737, 93 (2011)

[8] B. P. Abbott, et al., Astrophys. J. 713, 671 (2010)

[9] J. Abadie et al., Phys. Rev. D85, 022001(2012)

[10] J. Abadie et al., Phys. Rev. D85, 022001 (2012)

[11] B. Abbott et al., Nature 460, 990 (2009)

[12] J. Abadie et al., Phys. Rev. D85, 122001 (2012)

[13] J. Abadie et al., Phys. Rev. D85, 082002 (2012) 
[14] J. Aasi, et al., Phys. Rev. D87, 022002 (2013)

[15] J. Abadie et al., Phys. Rev. D85, 122007 (2012)

[16] C. D. Ott, Class. Quantum Grav. 26, 063001 (2009)

[17] K. N. Yakunin, P. Marronetti, A. Mezzacappa, S. W. Bruenn, C.-T. Lee, M. A. Chertkow, W. R. Hix, J. M. Blondin, E. J. Lentz, O. E. Bronson Messer, and S. Yoshida, Class. Quantum Grav. 27, 194005 (2010)

[18] W. Del Pozzo, T. G.F. Li, M. Agathos, C. Van Den Broeck, Phys. Rev. Lett. 111, 071101 (2013)

[19] S. E. Woosley, J.S. Bloom, Ann. Rev. Astron. \& Astrophys. 44, 507 (2006)

[20] R. C. Duncan, C. Thompson, Astrophys. J. 392, L9 (1992)

[21] J. Abadie et al., Astrophys. J. 734, L35 (2011) 